4 Chudley AE, Bauder F, Ray M, McAlpine PJ, Pena SD, Hamerton JL. Familial mental retardation in a family with an inherited chromosome rearrangement. $J$ Med Genet 1974;11:353-62.

5 Verjaal M, De Nef J. A patient with a partial deletion of the short arm of chromosome 3. Am J Dis Child 1978; $132: 43-5$.

Requests for reprints to $\mathrm{Dr} M$ de Arce, Genetics Division, Department of Pathology, University College, Earlsfort Terrace, Dublin 2, Ireland.

\section{Familial pericentric inversion of chromosome 11 detected prenatally}

SUMMARY A pericentric inv(11)(p12q25) was detected by prenatal diagnosis and subsequently found in four other family members. There was no apparent evidence of clinical consequences caused by this inversion.

With the advent of banding, pericentric inversions have now been described for the majority of human chromosomes. ${ }^{1}$ The most common inversion is a pericentric inversion of chromosome 9 , which occurs in approximately 1 to $1.5 \%$ of the population. ${ }^{23}$ Inversions of chromosome 11 are rarely seen, for generally this chromosome appears to be relatively stable. To our knowledge, there have been four cases of pericentric inversion of chromosomes 11, each having different breakpoints. ${ }^{4-6}$ This report

I

II
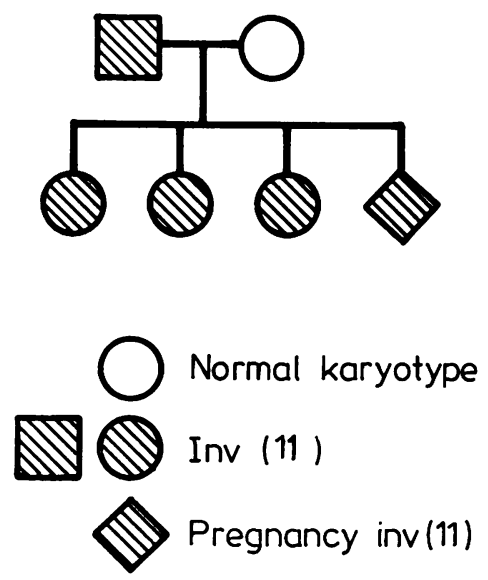

FIG 1 Pedigree of the family.

Received for publication 5 June 1981 describes a case of inv(11)(p12q25) that was shown to be present in two generations.

\section{Case report}

The proband, a 37-year-old Caucasian, gravida 4, para 3, and her 38-year-old spouse were referred for genetic counselling and prenatal diagnosis because of advanced maternal age. The pedigree is shown in fig 1 . There was no known family history of consanguinity, spontaneous abortions, or perinatal

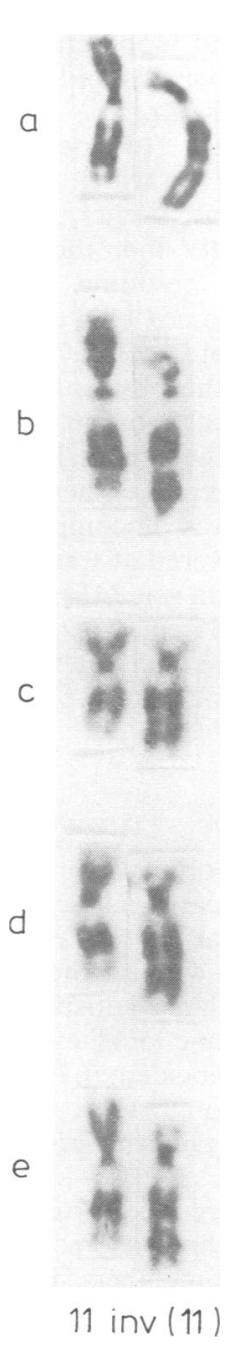

FIG 2 Partial karyotypes of (a) father, (b) fetus. and (c-e) sibs showing the pericentric inversion of chromosome 11. 
deaths. The three daughters were healthy with no apparent congenital anomalies.

\section{INVESTIGATIONS}

Amniocentesis and fetal ultrasound examination were performed in the 16th week from the last menstrual period. The ultrasound study and amniotic fluid $\alpha$-fetoprotein (17 $520 \mathrm{ng} / \mathrm{ml}$ by radioimmunoassay) were normal.

Amniotic fluid cells were cultured by standard techniques. Chromosome analysis after trypsinGiemsa banding revealed a pericentric inversion of chromosome 11 (46,XY,inv(11)(p12q25)). This structural rearrangement was shown to be paternal in origin after peripheral blood studies were done on both parents. All three sibs had the same inversion of chromosome 11 (fig 2).

Current knowledge of pericentric inversion carriers in a family in which segregation of the inverted chromosome occurs without producing recombinants indicates that the risk for clinically abnormal offspring resulting from duplicationdeficiency chromosomes is nominal, with an approximate risk of $1 \%{ }^{78}$ Based upon these findings, and upon the observation that the father and sibs were apparently normal, it appeared that the fetus was not at significantly high risk for congenital anomalies. The parents elected to continue the pregnancy, which was uncomplicated. A healthy male infant was delivered at term with no apparent anomalies. Birthweight was $3414 \mathrm{~g}$, length $52 \mathrm{~cm}$, and head circumference $35 \mathrm{~cm}$. The baby had an Apgar score of 8 at 1 minute and 7 at 5 minutes. The cord blood study confirmed the prenatal cytogenetic findings of inversion 11.

\section{Discussion}

As reviewed by Moorhead, ${ }^{7}$ inversions themselves do not appear to cause clinical anomalies. Production of duplication-deficiency chromosomes after crossing over within the inverted segment rarely results in unbalanced offspring or spontaneous abortions. Non-disjunctional events reported with inversions may be coincidental. Infertility in inverted carriers is rare although there has been questionable association with pericentric inversion of chromosome 9.

These findings are supported by the clinical cases of inversion chromosome 11 as well as our report of five family members. For inv(11)(p15q23) and (p11q11) described by Simola et al, ${ }^{4}$ there appeared to be no detectable clinical consequences related to either inverted chromosome, both of which segregated in three generations. Similar observations were reported by Autio-Harmainen and de la Chapelle ${ }^{6}$ for inv(11)(p11q13.3) and Boué and Boué ${ }^{5}$ for inv(11)(p15q14).

There is a general impression that unbalanced offspring are more likely to occur when a large part of the genetic material is inverted, leading to a greater incidence of crossing over and probable abnormal recombinant chromosomes. ${ }^{1}$ Although in the present case of inv(11)(p12q25) and in the case of Simola $e t a l^{4}$ of inv(11)(p15q23), where large pericentric inversions were segregating, there appeared to be little risk of abnormal offspring or spontaneous miscarriages in either family.

The work was supported by grant MCT-000423-17. We are grateful to Geeta Joseph, Charles Garner, Jesse Gore, and Barbara Myers for their technical assistance.

\section{Marcia H Russell, Phyllis Miller, and Allen Killam Division of Genetics, Department of Pediatrics, and Department of Obstetrics and Gynecology, Vanderbilt University Medical Center, Nashville, Tennessee, USA}

\section{References}

1 Winsor EJT, Palmer CG, Ellis PM, Hunter JLP, Ferguson-Smith MA. Meiotic analysis of a pericentric inversion, inv(7)(p22q32), in the father of a child with a duplication-deletion of chromosome 7. Cytogenet Cell Genet 1978;20:169-84.

2 Mutton DE, Daker MG. Pericentric inversion of chromosome 9. Nature 1973;241:80.

3 de la Chapelle A, Schroder J, Stenstrand K, et al. Pericentric inversion of human chromosomes 9 and 10 . Am J Hum Genet 1974;26:746-66.

4 Simola K, Karli P, de la Chapelle A. Two pericentric inversions of human chromosome 11. J Med Genet 1977;14:371-4.

5 Boué J, Boué A. Prenatal diagnosis in 100 structural rearrangements of the chromosomes. Cytogenet Cell Genet 1978;20:213-25.

6 Autio-Harmainen $\mathrm{H}$, de la Chapelle A. High resolution of a small pericentric inversion of chromosome 11 . J Med Genet 1980;17:44-7.

7 Moorhead PS. A closer look at chromosomal inversions. Am J Hum Genet 1976;28:294-6.

8 Sutherland GR, Gardiner AJ, Carter RF. Familial pericentric inversion of chromosome 19, inv(19)(p13q13), with a note on genetic counselling of pericentric inversion carriers. Clin Genet 1976;10:54-9.

Requests for reprints to Dr Marcia H Russell, Division of Genetics, Department of Pediatrics, Vanderbilt University Medical Center, Nashville, Tennessee 37232, USA. 